\title{
The current state of cancer genetic counseling access and availability
}

\author{
Sara Knapke, MS, LGC ${ }^{1}$, Joy Larsen Haidle, MS, CGC², Rebecca Nagy, MS, LGC ${ }^{3}$ and \\ Sara Pirzadeh-Miller, MS, CGC ${ }^{4}$
}

Purpose: Genetic risk assessment and counseling by a qualified genetics professional are recommended to ensure high-quality care for individuals at risk of hereditary cancer. Timely access to genetic services provided by a genetic counselor (GC) is essential, especially in cases where genetic testing results may affect impending surgical decisions.

Methods: A survey of GCs who specialize in cancer genetics was performed to assess service delivery models and ability to accommodate urgent cases.

Results: Over half of all respondents indicated that urgent patients can be seen for consultation the same day or within 1-2 business days, and almost all respondents indicated that urgent cases can be seen within 1 week. Most respondents indicated that urgent cases are seen by a GC only with no physician involved.

Conclusions: The results of this survey of GCs demonstrate that timely access to cancer genetic counseling by GCs in an urgent setting is available.

Genet Med advance online publication 6 August 2015

Key Words: access; cancer genetics; cancer risk assessment; genetic counseling; genetic testing
A 42-year-old woman was recently diagnosed with triple-negative breast cancer. Her family history includes a paternal aunt with breast cancer in her fifties, a paternal grandmother with ovarian cancer in her sixties, and paternal first cousins with pancreatic and early-onset colorectal cancers. She is facing a surgical decision to pursue lumpectomy or bilateral mastectomy. Is timely genetic counseling and genetic testing available to help her with her decision?

Genetic risk assessment and counseling by a qualified genetics professional has been outlined as an essential standard by the American College of Surgeons Commission on Cancer ${ }^{1}$ and the National Accreditation Program for Breast Centers. ${ }^{2}$ In addition, there is research demonstrating patient harm in the absence of high-quality genetic services accompanying genetic testing. ${ }^{3-6}$ However, perception of limited access to and availability of cancer genetic counseling services by a board-eligible or board-certified genetic counselor (GC) has the potential to form an unnecessary barrier to comprehensive genetic risk assessment, counseling, and testing. In this report we outline the current state of access to genetic counseling, including evidence from a recent survey of GCs focused on wait time for urgent consultations.

Recent data from the National Society of Genetic Counselors (NSGC) Professional Status Survey (PSS) demonstrates that access to GCs is excellent and in line with access to non-genetics physicians. The PSS "provides a detailed profile of the [NSGC] membership and identifies new and emerging trends in the genetic counseling profession." The 2014 PSS was administered to members of the NSGC and to diplomates of the American Board of Genetic Counselors from 13 January through 26 February 2014. Nearly half (46\%) of the GCs who responded to this section of the PSS $(N=1,409)$ report that they are able to accommodate a new patient for consultation within 1 week; $27 \%$ report that they could see a new patient within 1-3 days. Wait time for a consultation was significantly longer for patients who were seen by both a GC and a physician. ${ }^{7}$ Of note, cancer genetic counseling is most likely to involve an exchange of information regarding the risks, benefits, and limitations of genetic testing and facilitation of genetic testing as needed; therefore, GCs are well within their scope of practice to provide cancer genetic services to patients without a coupled physician encounter.

There is also marked growth in the number of GCs practicing in the United States. Since 2006, there has been a $75 \%$ increase in the workforce and, with a growing number of training programs and expanded capacity within existing training programs, the annual growth in the profession is expected to be upward of $7-10 \%$. There are currently more than 3,500 certified GCs. ${ }^{8}$ Of the GCs who participated in the 2014 PSS, nearly $30 \%$ consider cancer genetics their primary specialty. In addition,

${ }^{1}$ GeneDx, Elmwood Park, New Jersey, USA; ${ }^{2}$ Guardant Health, Redwood City, California, USA; ${ }^{3}$ Humphrey Cancer Center, Minneapolis, Minneapolis, USA; ${ }^{4}$ Simmons Cancer Center, University of Texas South Western Medical Center, Dallas, Texas, USA. Correspondence: Sara Knapke (sknapke@genedx.org)

Submitted 3 February 2015; accepted 28 May 2015; advance online publication 6 August 2015. doi:10.1038/gim.2015.98 
$74 \%$ of respondents reported that they counseled patients as a regular part of their jobs. ${ }^{7}$

In addition to face-to-face consultations, innovative service delivery models such as genetic counseling via telephone or video conferencing have significantly increased access for patients in rural and previously underserved areas. In fact, patients making surgical decisions based on genetic information who need expedited services are often able to access telephonic services within a day (A. Trivedi, personal communication).

To gather access and availability data specific to urgent cancer genetics cases, cancer GCs were recently surveyed about their staffing approach within the in-person, telegenetic, or telephonic service delivery models and ability to accommodate an urgent appointment (defined as a genetic test that result would affect immediate surgical/treatment decision-making for a patient newly diagnosed with breast cancer).

\section{MATERIALS AND METHODS}

The survey was conducted by the NSGC. Institutional review board approval was not obtained or required. A survey was sent via email by the NSGC executive office to members of the NSGC Familial Cancer Special Interest Group. Data collection occurred in May 2014. The survey included 11 questions regarding wait times for an urgent patient to see a GC and determined whether these wait times varied depending on staffing approach within the in-person, telegenetic, or telephonic service delivery model settings. These options include the patient seeing a GC only or the patient seeing a GC and a physician. The patient scenario used was "a newly diagnosed breast cancer patient using the results of the genetic test to make decisions about lumpectomy vs. mastectomy." The survey was sent to a total of 691 NSGC members, of whom 334 members participated (a 48\% response rate). Members received an invitation to participate in the survey, one reminder e-mail, and two online discussion forum posts over the course of 8 days. The Web link to the survey was inactivated at the end of the data collection period. Respondents' identities were not known, and no personally identifiable information was linked to the survey responses. The submission of a completed survey questionnaire signified informed consent to participate.

\section{RESULTS}

Regarding their staffing approach within the service delivery models, the majority (56\%) of the 334 survey participants
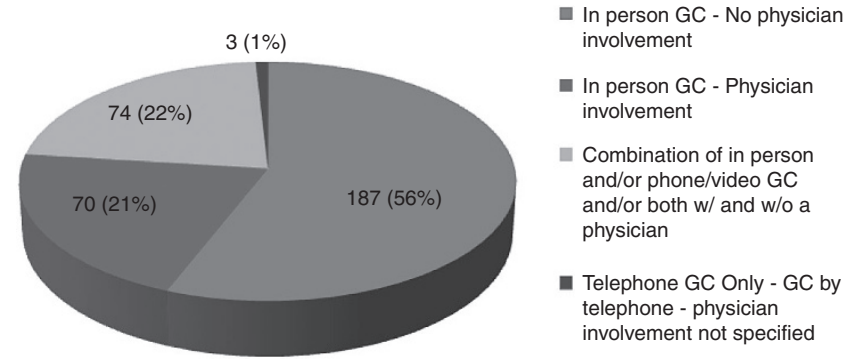

- Telephone GC Only - GC by telephone - physician involvement not specified

Figure 1 Genetic counseling service delivery models and staffing for urgent patients $(n=334)$. indicated that patients are seen by a GC in consultation without a physician. A near equal number of respondents indicated that they either see patients with a physician $(21 \%)$ or use a combination provider approach $(22 \%)$ in their program. Less than $1 \%$ of respondents indicated that they use a telephonic service delivery model only (Figure 1).

For those who indicated use of a combination provider approach $(n=74)$, open-ended responses elucidate that many GCs see some patients independently and some patients with a physician; others describe that some patients are seen in person, and other patients are counseled by phone or video conference. Reported reasons for coupling or decoupling the provider approach for patients include whether the consultation is a new or follow-up visit, whether results of the genetic testing were negative or positive, and whether patient insurance covers the cost of a GC-only visit. Of note, a number of participants indicated that they use a combination provider approach and triage patients to see a GC only if the need is urgent. Specifically, nearly $80 \%$ of those respondents who use a combination provider approach indicate that the wait time for an urgent appointment is shorter for a GC-only visit.

Of respondents who use a GC only or GC/MD provider approach $(n=268), 96 \%$ indicated that they could see an urgent patient within 7 business days. More specifically, $61 \%$ of respondents reported wait time of less than 2 business days (Table 1 ). Only $3.7 \%$ of respondents reported wait times of more than 1 week for urgent referrals. Of the GCs, $94-98 \%$ report that wait times for a GC-only visit are shorter than for patients needing to see both a GC and MD. When asked how urgent referrals ensure expedited care, most replied that "urgent appointment slots" are held daily at their institution, or their centers adjust the GC's schedule to accommodate the patient into the existing schedule. To further increase access for an urgent consultation, over half of all respondents (53\%) indicated that if the wait time at their center was longer than 7 days, they would refer the patient to another GC who could see the patient more quickly.

\section{DISCUSSION}

The results of this survey indicate that there is adequate availability of genetic risk assessment, counseling, and testing with a GC in an urgent setting. Sixty-one percent of respondents indicated appointment wait times of fewer than 2 business days for urgent patients, and $96 \%$ of respondents could facilitate urgent appointments in fewer than 7 business days. The diversification of service delivery models in genetics will also allow cost-effective and sustainable avenues to meet the ever-growing demand for genetic counseling services. ${ }^{9}$ Significant annual

Table 1 Genetic counselor reported wait time for urgent patients $(n=268)$ Respondents (\%) 
increases in the numbers of GCs continues to alleviate concerns of a professional shortage.

Other factors may impose barriers to services with a GC. Examples include perceived or actual lack of insurance coverage for genetic services and/or genetic testing, competing priorities, and patients who self-select out of such services. In addition, underuse of telephonic genetic counseling services, particularly in more rural regions without access to other genetic service delivery models, affect the perceived concerns about access to GCs. Continued education efforts of health-care providers in regards to the multiple service delivery models available to their practice, and patients could modify this perception. Further study is needed to characterize these barriers, and it is imperative that the health-care team work together to address the barriers to the betterment of patient care and outcomes.

Not all cancer GCs are members of the NSGC or subscribe to the discussion forum, which limits the possibility of maximum feedback. The time allowed for participation was quite short, which also could have limited participation. Finally, these data are self-reported, which allows for the possibility of bias; future studies on the topic could utilize feedback outside of the cancer GC community. Future studies could also examine the efficiency of multiple service delivery models and the availability of these per geographic service area to identify any specific underserved regions. Data such as these could help to alleviate potential genetic service access issues in any identified areas.

In summary, barriers to timely genetic counseling, risk assessment, and testing by a GC have decreased substantially as a result of a growing number of GCs and services delivery models that allow GCs to accommodate patients quickly.

\section{ACKNOWLEDGMENTS}

The authors acknowledge the members of the Cancer Special Interest Group and the Executive Office of the NSGC.

\section{DISCLOSURE}

S.K. is an employee of GeneDx. J.L.H. is a consultant for Blue Cross Blue Shield of Minnesota. The other authors declared no conflict of interest.

\section{REFERENCES}

1. Commission on Cancer. Cancer Program Standards 2012: Ensuring PatientCentered Care. https://www.facs.org/quality-programs/cancer/coc/standards. Accessed 3 February 2015.

2. National Accreditation Program for Breast Centers. NAPBC Standards Manual 2014. https://www.facs.org/quality-programs/napbc. Accessed 3 February 2015.

3. Bonadies DC, Brierley KL, Barnett RE, et al. Adverse events in cancer genetic testing: the third case series. Cancer J 2014;20:246-253.

4. Brierley KL, Blouch E, Cogswell W, et al. Adverse events in cancer genetic testing: medical, ethical, legal, and financial implications. Cancer J 2012;18:303-309.

5. Brierley KL, Campfield D, Ducaine W, et al. Errors in delivery of cancer genetics services: implications for practice. Conn Med 2010;74:413-423.

6. Bensend TA, Veach PM, Niendorf KB. What's the harm? Genetic counselor perceptions of adverse effects of genetics service provision by non-genetics professionals. J Genet Couns 2014;23:48-63.

7. National Society of Genetic Counselors Professional Status Survey, 2014. http:// www.nsgc.org.

8. American Board of Genetic Counseling. http://www.abgc.net

9. Cohen SA, Marvin ML, Riley BD, Vig HS, Rousseau JA, Gustafson SL. Identification of genetic counseling service delivery models in practice: a report from the NSGC Service Delivery Model Task Force. J Genet Couns 2013;22:411-421. 